Revue d'histoire de l'Amérique française

ZRS REVUE D.HISTOIRE DE L'AMÉRIQUE FRANÇAISE

\title{
Chronique des sources privées de l'histoire coloniale
}

\section{Roger Massio}

Volume 8, numéro 1, juin 1954

URI : https://id.erudit.org/iderudit/301645ar

DOI : https://doi.org/10.7202/301645ar

Aller au sommaire du numéro

Éditeur(s)

Institut d'histoire de l'Amérique française

ISSN

0035-2357 (imprimé)

1492-1383 (numérique)

Découvrir la revue

Citer ce document

Massio, R. (1954). Chronique des sources privées de l'histoire coloniale. Revue d'histoire de l'Amérique française, 8(1), 139-153.

https://doi.org/10.7202/301645ar d'utilisation que vous pouvez consulter en ligne.

https://apropos.erudit.org/fr/usagers/politique-dutilisation/ 


\section{BIBLIOGRAPHIE}

\section{CHRONIQUE DES SOURCES PRIVEES DE L'HISTOIRE COLONIALE}

Comme l'a indiqué M. Debien ${ }^{1}$, les archives coloniales privées sont, en ce qui concerne Saint-Domingue, d'une extrême richesse. Mais elles sont très dispersées, car toutes nos provinces ont envoyé des colons aux iles et entretenu avec elles des correspondances. Ces papiers sont surtout des papiers de famille ou des papiers d'affaires: ils ont trait, directement ou indirectement, à l'histoire des plantations, à leurs origines et aménagement, à leur main-d'œuvre, à la marche des cultures, de la production et des revenus. C'est ainsi que peuvent être éclairés quelques-uns des plus importants problèmes que pose l'histoire générale des Antilles.

\section{Les papiers Navailles-SÉguineau $(1745-1829)^{2}$}

Le Béarn et la Bigorre ont envoyé des colons à Saint-Domingue, ont eu des propriétaires de plantations. Des papiers de ces colons et de ces anciens propriétaires dorment depuis plus d'un siècle dans quelques vieux greniers. Le hasard parfois les ont fait sortir. C'est ainsi qu'un lot de vieux papiers, au moment de la vente de l'immeuble d'Olce, à Vic-en-Bigorre fut confié à M. Gardey, curé de Vic. Avant leur dépôt aux Archives des Hautes-Pyrénées, M. Gardey a eu la grande obligeance de nous laisser mettre à profit ces documents. Ils viennent de la famille Navailles et de sa caféière du quartier de

1. G. Debien, "Archives de Plantation des Antilles," in Bulletin Sté Arch. de Nantes, (1946), T. 85.

2. Cf. R. Massio, "Les papiers Séguineau-Navailles" (1745-1829), Rev. d'hist. des Colonies, 3e et $4 \mathrm{e}$ trim. 1950. 
Montrouy à Saint-Domingue. Ils ont dû être importants et en bon ordre, mais nous n'en avons plus que des épaves et peu cohérentes. Cependant, même ainsi réduites, ces liasses présentent un grand intérêt. Elles comprennent:

10 - des actes d'état-civil, puis des testaments et des arrangements de famille. Nous voyons au XVIIIe siècle l'alliance d'une Navailles avec un Séguineau, de Bordeaux, propriétaire "aux isles". Par la suite, Navaille-Bonnas, conseiller au Parlement de Pau, héritera par sa femme, née Séguineau, de la plantation de SaintDomingue. On peut suivre, au moins en partie l'évolution sociale d'une famille. On touche ici au négoce bordelais, par les Séguineau, au milieu parlementaire de Pau et rural de Bigorre, par les Navailles.

20 - Un compte de gestion de caféière, de 1788, particulièrement suggestif, quelque partielles que fussent les conclusions qu'on en pourra tirer. Nous avons d'importants comptes de sucreries, mais presque rien de la comptabilité des caféières. Navailles-Bonnas est un de ces maitres qui n'ont jamais vu leurs plantations. Elles ne représentent plus que des capitaux lointains, aux revenus mal contrôlés, donc très aléatoires et irréguliers.

3o-Une partie de la correspondance échangée entre les "procureurs de l'habitation", les frères Terrien, de Nantes et Navailles-Bonnas. Cette correspondance qui fut régulière, a dû être très abondante. Il ne reste plus que quelques lettres de 1790 , mais qui touchent à de nombreuses questions. D'abord, à la vie politique: 1790 est pour Saint-Domingue le moment où une partie des colons commence à se détacher de la France pour résister à sa politique favorable, bien timidement pourtant, à l'égalité des couleurs. Il est souvent parlé dans ces lettres du problème des mulâtres libres. Les Terrien exposent le point de vue des blancs et marquent bien la réaction du grand commerce devant la politique autonomiste de l'assemblée coloniale. Leurs lettres offrent ainsi quelques éléments pour une carte des opinions politiques dans la colonie.

$\mathrm{Au}$ sujet des esclaves, nous apprenons peu de chose. Il nous faudrait des comptes et des lettres s'échelonnant sur plus d'années. Et les lettres des procureurs sont généralement des témoignages dirigés. Elles doivent être examinées avec prudence. Mais la conclusion des Terrien devant la division des blancs est pleine d'un bon sens qui voit loin. 
La correspondance des Terrien éclaire aussi des problèmes agricoles. Elle insiste sur la nécessité des fumures, sur les sols vite épuisés ou détériorés. Les terrains consacrés aux caféiers sont des terrains récemment défrichés et de bon rapport. Il s'agit de conserver le plus longtemps possible les vertus fertilisantes d'un sol aux brusques inconnues.

4o - Quelques lettres enfin disent ce que fut l'administration générale des biens d'absents au temps de l'occupation anglaise, de 1794 à 1797 , et comment fut réglée, après 1826, la question de l'indemnité accordée par l'ordonnance royale qui reconnaissait l'indépendance de l'ancienne colonie. Et quand la perte de la caféière a réduit leur fortune, quand celle de Saint-Domingue, définitive en 1825, a fait tomber leurs derniers espoirs coloniaux, on voit, à travers ces papiers, s'opérer pour les Navailles-Bonnas, ce rétrécissement d'horizon économique et social qui fut une des conséquences les plus durables, bien qu'une des moins apparentes de la Révolution.

Ce que l'émigration et le retour au milieu des biens nationaux vendus a produit chez tant d'autres, la ruine de Saint-Domingue l'a fait chez les Navailles-Bonnas. Elle a provoqué pour plus d'un demi-siècle cet enracinement de la petite noblesse et préparé son influence politique et sociale, si grande dans certaines régions de la France rurale du XIXe siècle ${ }^{3}$.

\section{Les papiers Cestia}

Pour la Guadeloupe, on a grand'peine a trouver des correspondances privées, des dossiers de plantation, des papiers de commerce et ils sont toujours fragmentaires. C'est comme pour la Guyane et la Martinique, cependant si pauvres. Et ce qui est vrai des XVIIe et XVIIIe siècles, l'est davantage encore du XIXe siècle, le siècle partout de la documentation difficile. Nous sommes donc heureux de dire quelques mots des papiers d'affaires de la Guadeloupe, abordée cet hiver.

3. R. Massio, "Un dossier de plantation" (1745-1829), Revue d'histoire de l'Amérique française, V, no 4 (Mars 1952): 542-577 et vol. VI, no 1 (juin 1952): 52-109. 
Près de Vic-en-Bigorre, sur la route de Tarbes, est le domaine de Saint-Onis. Au XVIIIe siècle, y vivait noble Eugène de Lataste, seigneur du Pouey. Si on ne peut assurer sa parenté avec les Lataste du nord de Saint-Domingue, du moins le mariage de sa fille, Marie, en 1739, avec Pierre de Thèze, colon au Petit-Trou, montre SaintOnis orienté vers les Antilles dès le XVIIIe siècle.

Le domaine fut vendu, en 1823 par la dernière Lataste, à Jean Brescons, planteur à La Lézarde, quartier du Petit-Bourg, à La Guadeloupe, qui décéda en 1826, à Pointe-à-Pitre. Il dut laisser une situation embarrassée, car sa plantation fut vendue par licitation, au profit de René Claudin et SaintłOnis, passa au même moinent vers 1830, à Bertrand Cestia, négociant à Pointe-à-Pitre. Il est probable que Brescons et Cestia étaient d'origine bigourdane. Par héritage, Saint-Onis, vint des Cestia aux Bordères, de Vic-en-Bigorre, qui l'ont toujours.

Dans le grenier de Saint-Onis, sont trois malles pleines à craquer de vieux papiers. Une courte après-midi d'hiver a permis de vider l'une d'elles. On ne peut même pas parler d'exploration. Tout est à classer. Mais ce premier coup d'œil a fait distinguer:

10 - un gros dossier de 4 à 500 lettres reçues à Pointe-à-Pitre, par la maison Cestia, de 1823 à 1830, toutes d'intérêt commercial.

20 - Un cahier de copie de lettres, expédiées de Pointe-à-Pitre, par la maison Cestia, du 28 mars 1827 au 14 août 1828. Il a 101 pages et compte environ 150 lettres, adressées à des colons de la Guadeloupe, à des commerçants des îles voisines (Saint-Thomas et Porto Rico), à des maisons bordelaises: Despalangues, Laville, Martin, Estiboy, Turettes et Bordères; à des maisons du Havre. Les affaires principales sont avec Bordeaux: achats de vins, cognacs, vinaigres, fromage, confection pour les esclaves, maĩs, farines et surtout morues.

On remarque d'abord les lettres adressées à la maison Despalangues, au sujet d'une certaine "exploration de la côte d'Afrique"; par le brick l'Éclair, armé à Saint-Thomas, en octobre 1827, entendez au sujet d'une expédition négrière. Le bâtiment de 8 canons et de 25 hommes d'équipage, dojt ramener 350 esciaves. Jamais on ne lit le mot esclaves. Non pour se mettre "à l'abri de toute recherche en cas d'événements", mais par une sorte de pudeur de style, qui tranche avec le reste qui est en clair. Le retour est attendu pour 
janvier ou février 1828. La mise en état du bâtiment monte à 130.000 francs: état du bâtiment 60.000 francs pour le corps du navire; 70.000 pour la cargaison d'achat. L'assurance est de $30 \%$, on ne dit point le tonnage.

Précédemment, 312 esclaves avaient été traités en quinze jours, 110 autres même en cinq jours. Cela nous donne une idée de la minutieuse préparation et du choix méthodique des sites de traite. $\mathrm{Au}$ XVIIIe siècle, la traite libre était une lente quête de captifs d'un port à l'autre. Au XIXe siècle, la traite surveillée, interdite, s'est organisée comme un rapide enlèvement. L'Éclair est capturé le 2 février 1828 par une croisière française. Les captifs étaient encore à terre. Il fallut envoyer des îles un nouveau navire vide, qui ramena 300 esclaves au lieu des 350 prévus. On se réjouit du résultat.

Le sondage est donc fort prometteur. Il propose une entreprise de longue haleine: l'histoire d'une maison de commerce de la Guadeloupe et de ses rapports avec Bordeaux et avec la côte d'Afrique, aux dernières années de la Restauration.

\section{$*_{*}^{*}$ \\ Les Papiers LarcheVesque-Thibaud}

La découverte de sources privées est bien souvent le fait du hasard. Ainsi, des papiers de la famille Larchevesque-Thibaud, pieusement conservés, de génération en génération, sont arrivés en nos mains. La personne ayant bien voulu nous confier ces papiers, désirant conserver l'anonymat, nous ne pouvons indiquer la provenance de ces documents. L'esprit de famille a ses pudeurs, il faut les respecter. Cependant, d'après les mentions portés sur certains actes, il est permis de soupposer que ces archives familiales furent conservées par l'arrière-petite-fille du constituant LarchevesqueThibaud. Cette transmission de souvenirs familiaux montre, contrairement à ce que certains pensent, que la bourgeoisie n'a pas la mémoire courte et que le culte des ancêtres se maintient. Rendons donc un témoignage de reconnaissance à ceux qui ayant le goût du passé ont conservé ces vieux papiers.

Jean-Baptiste LARCHEVESQUE-THIBAUD était né a Larcahaye, à Saint-Domingue, en 1745. Il était le fils de Gabriel Lar- 
chevesque-Thibaud et de Marguerite Violain. La famille était propriétaire d'une très importante caféière au quartier de Vallière dans le nord de l'île. Le 27 janvier 1789, les électeurs de toutes les paroisses dont la ville du Cap était le chef-lieu, désignèrent Larchevesque-Thibaud avec quinze autres planteurs comme représentant de la partie nord de l'île aux États Généraux. Mais Larchevesque-Thibaud démissionnait dès le 24 août 1789. Il devait faire partie de l'assemblée du Nord, puis de l'Assemblée de Saint-Marc et des 85. Resté en France, après le départ de la plupart de ses collègues, il avait agi contre le décret du 15 mai et, de retour à Saint-Domingue, il était devenu procureur de la commune du Cap. Les historiens ont coutume de représenter Larchevesque-Thibaud comme un grand agitateur de la colonie. Il est vrai qu'il fut particulièrement actif contre le gouverneur Blanchelande. De même, en 1792, il travailla d'accord avec Polverel et Sonthonax, puis s'étant séparés d'eux, les commissaires civils le déportèrent en France où il poursuivit son action contre eux, afin d'obtenic leur mise en accusation. D'autre part, en 1793, il fut désigné pour faire partie d'une délégation par les assemblées que les "colons patriotes" réfugiés avaient formée aux Etats-Unis. Il est poursuivi le 18 messidor, an II, par Fouquier, accusateur public qu'il appelait son "cher ami", devant le tribunal révolutionnaire. Il avait été arrêté en même temps que d'autres colons, le 19 ventôse, an II. Il était accusé d'être complice du projet d'assassiner les gardiens de la maison d'arrêt et les membres du Comité de Salut-Public et de sûreté générale et autres patriotes. Il fut détenu à la maison d'arrêt de la Conciergerie. Il avait alors quarante-neuf ans. Puis, par jugement du tribunal révolutionnaire qui le reconnut innocent des griefs qui lui étaient imputés, en date du 22 messidor, an II (10 juillet 1794), sur nouveau réquisitoire de l'accusateur public et pour "autres causes et délits, "dont la connaissance n'est point attribuée à ce tribunal, il fut transféré à la maison du Luxembourg dont les portes lui furent ouvertes providentiellement le 10 thermidor. Alors, comme les autres commissaires désignés par les assemblées de Philadelphie, Baltimore, New-York, Larchevesque-Thibaud continua à mener campagne contre Polverel et Sonthonax... Comme la plupart des commissaires, il soutenait l'autonomie coloniale, la liberté du commerce, le maintien de l'esclavage. La commission des colonies tint séance le 30 janvier 1795 . 
Elle reconnut le 7 février 1795 et agréa Larchevesque-Thibaud, mais d'après Garran-Coulon (rapport IV, p. 596) pendant les débats, Larchevesque se retira parce que "la commission ne crut pas devoir laisser un libre cours à ses divagations".

Le Directoire, le Consulat voient Larchevesque toujours préoccupé de Saint-Domingue et espérant une restauration prochaine de la souveraineté prochaine dans l'île. En 1803, à nouveau attiré par les Antilles, il quitte la France pour venir se fixer à la Guadeloupe, comme avocat et avoué près la Sénéchaussée de Pointeà-Pitre. Il est recommandé par son ancien collègue à la Constituante, $\mathrm{Mr}$ de Talleyrand, alors ministre des relations étrangères, par deux lettres du 3 et 12 floréal, an IX (23 avril et 2 mai 1803). Il meurt à 72 ans, en 1817, à Pointe-à-Pitre.

Larchevesque-Thibaud, de son mariage, avec Louise-Catherine de Castonnet-Desfosses, native de Saint-Domingue, avait eu quatre enfants: deux fils et deux filles. L'aîné, Pierre-Louis-Gabriel, né le 17 août 1787, mort en 1844, à 57 ans, à Pointe-à-Pitre, fut avoué dans cette ville. Le second, Jean-Baptiste, né en 1792, au Cap Français, mort en 1852, à Lorient âgé de 60 ans, fut ingénieur de la Marine, à La Martinique, à la Guadeloupe et aux Saintes. Les deux filles du Constituant se marièrent, l'une à un certain Cranfon, l'autre à Néron de Verville. Elles sont les tantes de la personne qui a conservé ces papiers et que nous supposons être une fille de l'avoué.

Pierre-Louis Gabriel épousa Anne Chastel, à Pointe-à-Pitre. Son beau-père possédait une plantation à La Guadeloupe, dans la commune du Morne à l'Eau, appelée "La Belle Espérance."

Ces détails sont indispensables pour comprendre l'intérêt des papiers de Larchevesque-Thibaud. En effet, les LarchevesqueThibaud viennent s'établir sous l'Empire à la Guadeloupe. Larchevesque-Thibaud père (une lettre dans le dossier est fort suggestive à cet égard) conserve encore l'espoir, en 1814, de rentrer en possession de son habitation de Vallière à Saint-Domingue. Le séjour à La Guadeloupe n'est dans son esprit que momentané. Il semble avoir des renseignements sur la question coloniale par ses anciennes et puissantes relations. Parmi ces dernières, il faut compter: Talleyrand, son ancien collègue à la Constituante, Malouet, autre collègue du début de la Révolution et fort au courant des choses de Saint- 
Domingue; François de Neufchâteau, un certain M. de Nazon, ami intime et qui fut aussi propriétaire à Saint-Domingue. Mais il meurt en 1817, avant d'avoir vu régler la question de SaintDomingue. C'est à ce moment que son fils, avoué à Pointe-à-Pitre, va de sa propre main, recopier certains documents intéressant l'activité de son père à la veille de la Révolution, à Saint-Domingue. Il semble qu'il ait voulu constituer un dossier familial préservant de l'oubli ou de la destruction certains papiers.

Ainsi figurent dans ces papiers de famille de la main de Larchevesque-Thibaud fils:

1 - Copie du procès-verbal de la nomination de Messieurs les députés de la partie du Nord de Saint-Domingue aux Etats Généraux de France, le 27 janvier 1789.

2 - Lettre de $\mathrm{M}^{\mathrm{r}}$. Larchevesque-Thibaud, député de l'assemblée générale de la partie française de Saint-Domingue, adressée à la municipalité du Cap.

C'est une des pièces les plus importantes de notre dossier. Il est question du décret du 8 mars, de l'indépendance de Saint-Domingue. Larchevesque-Thibaud se défend d'être favorable à l'indépendance, mais en réalité comme de nombreux colons blancs, il songe à l'autonomie. Il est aussi question de l'abbé Grégoire, des gens de couleur libres. Larchevesque affirme sa "fidélité inviolable au roi", mais comment concilier autonomie et fidélité ? Il traite d'imbéciles "ceux qui l'accusent d'être l'apôtre de l'indépendance". Et pourtant?

Cette lettre est datée de Saint-Marc, le 8 juin 1790; elle fut imprimée, dit une note, par ordre de Madame Larchevesque-Thibaud. Larchevesque fils qui semble recopier le texte imprimé, écrit: "pour copie conforme à l'imprimé qui est en mes mains. Pointe-àPitre, le (sans date), Larchevesque-Thibaud."

Si le document original a disparu des archives des colonies, cette lettre est d'un réel intérêt et mérite une publication intégrale car elle donne une vue très précise de la position politique prise par Larchevesque-Thibaud, en juin 1790. Est-ce la position d'un homme "toujours impulsif, fantasque, brouillon et incapable de se fixer", comme le note Garran-Coulon? (Rapport IV, p. 596). Si GarranCoulon juge ainsi Larchevesque-Thibaud, il semble que ce soit avec malveillance. 
3- Copie de l'acte d'accusation dressé par Fouquier contre Larchevesque-Thibaud (Juillet 1794).

4 - Copie de deux lettres "qui ont été écrites par Mr de Talleyrand à mon père les 3 et 12 Floréal, an IX" ( 23 avril et 2 mai 1801).

5 - Copie d'une lettre écrite à mon père par Mr Labiche de Reignefort, procureur du roi à la Cour d'Appel de la Guadeloupe, le 4 juillet 1814.

6 - Copie d'une lettre écrite à mon père, par $\mathrm{Mr}$ Butel de Montgai, lors président de la Cour Royale de la Guadeloupe, le 2 octobre 1815.

7 - Lettre de Larchevesque-Thibaud père, avocat à La Pointeà-Pitre, adressée le 9 septembre 1814 , à un ami $\mathrm{Mr}$ de Nazon, qui fut colon à Saint-Domingue, avant 1789. Cette lettre revêt une importance particulière, du fait qu'elle est écrite de la main même de l'ancien constituant et que, par conséquent, elle est inédite. On y voit Larchevesque-Thibaud compter sur un rétablissement de la situation à Saint-Domingue. Il est très affecté de la mort de Malouet, mais il compte sur M. de Nazon pour lui procurer la protection du nouveau ministre. La Guadeloupe est encore aux mains des Anglais. "La colonie doit être remise aux autorités françaises", sous peu, écrit Larchevesque-Thibaud et "tous les Français attendent ce moment avec impatience non que nous ayons à nous plaindre du gouvernement anglais..." Et pour cause! un de ses fils a été maintenu comme avoué par sir Alexandre Cochrane, gouverneur anglais de la Guadeloupe. Plus loin, l'ancien constituant fait part de son désir de retourner à Saint-Domingue sur sa plantation de Vallière. Il affirme que ses biens n'ont pas trop souffert et "qu'il ne faudrait pas cinq ans pour pouvoir me retirer en France avec une fortune honnête". Ses relations avec de nombreux membres de la Chambre des Pairs lui faciliteront toutes choses.

8 - Actes d'état-civil de la famille Larchevesque-Thibaud.

9 - Papiers relatifs à Larchevesque-Thibaud sls, ingénieur de la Marine. Jean-Baptiste Larchevesque-Thibaud prépare, en 1809 le concours d'entrée à l'École polytytechnique où il est admis en 1810, avec le numéro 43. Il sort de l'école, en 1812 et comme sous-lieutenant prend une part active au siège d'Anvers, puis passe à La Martinique. Aux Antilles, il fait exécuter de nombreux tra- 
vaux. Il indique qu'il a réuni au Fort National (Martinique) à son service d'ingénieur celui des Travaux hydrauliques. Il a organisé le service du curage après y avoir construit le matériel nécessaire pour le dévasement du port. Il a également construit un petit arsenal de la Marine pour exécuter les travaux relatifs à la flotte qui y est stationnaire et pour l'usage de la colonie, ainsi que plusieurs débarcadères.

A Saint-Pierre, il a relevé deux goélettes de l'Etat et plusieurs navires de commerce jetés à la côte, à la suite du coup de vent du 19 décembre 1832. A la Pointe-à-Pitre, Larchevesque-Thibaud a aussi organisé le service du curage. A la Basse-Terre, il a construit un embarcadère pour le service des bâtiments de la station. Aux Iles des Saintes, il a construit et réparé dans la rade des CorpsMorts pour le service des bâtiments de la station pendant l'hivernage. Enfin, les papiers signalent une mission d'exploration dans l'île de la Martinique où Larchevesque établit "trois lignes smaphoriques" (sic) lors de la Guerre d'Espagne, en 1823. En 1833, Larchevesque-Thibaud est de retour à Lorient où il terminera sa carrière après un séjour à Cherbourg, comme ingénieur de la Marine de 1ère classe.

10 - Papiers relatifs à Larchevesque-Thibaud, avoué à Pointeà-Pitre. Une partie de la carrière du fils aîné se déroule sous l'occupattion anglaise. Ainsi, "le 2 du mois d'août 1811 et la 51ème année du règne de sa Majesté, (britannique), son Excellence l'honorable sir Alexandre Cochrane, gouverneur de la Guadeloupe", nomme Larchevesque-Thibaud fils "avoué adjoint du Tribunal de première Instance de la Pointe-à-Pitre". La première nomination d'avoué titulaire, en date du 8 septembre 1814 est aussi faite par le gouverneur anglais sir John Skimmer. Ces deux nominations posent le problème de savoir dans quelle mesure Larchevesque-Thibaud père fut sous la Révolution à Saint-Domingue en excellent rapport avec les Anglais? Ne fit-il pas partie du groupe de colons de Saint-Domingue qui pensèrent un moment appeler les Anglais et peut-être leur livrer l'île? Ces deux nominations seraient un témoignage de reconnaissance envers le père, reporté sur le fils? Les émigrés royalistes sous la Révolution n'avaient-ils pas eu des contacts avec les Anglais au sujet de Saint-Domingue? Des contacts de même nature ont pu exister entre le Constituant et les Anglais. Toujours est-il que 
la Commission d'avoué est confirmée lorsque la Guadeloupe redevient française par le comte de Linois, gouverneur de l'île, en 1814 et en 1815 .

De 1811, à 1832, Larchevesque-Thibaud fils remplira ses fonctions d'avoué à la Pointe-à-Pitre. En 1832, il demande un congé pour se rendre en France. Il l'obtiendra en 1834. Il s'embarque à cette date, avec son épouse, ses huit enfants et "la nommée Angèle, mulatresse âgée de 48 ans".

11 - Dossier Chastel. Plan de l'habitation Chastel (sucrerie, située au Morne-à-l'Eau, Guadeloupe). Dans ce dossier figure un plan de l'habitation Chastel appelée "La Belle Espérance". C'était une sucrerie. On y distingue les différentes parcelles avec le jardin potager, la maison principale, le moulin à vent, le moulin rond, la sucrerie à purgerie avec sa cheminée, un parc à bœufs, le pavillon de l'économe, l'emplacement des cases à nègres, type de sucrerie assez semblable à celles qui existaient à Saint-Domingue au 18ème siècle et dont le $\mathrm{P}$. Labat nous a donné d'abondantes descriptions. Cette sucrerie appartenait au beau-père de Larchevesque-Thibaud fils qui avait épousé Anne Chastel.

Dans le même dossier se trouvent des papiers concernant un fils Chastel, Chastel Pierre, chirurgien et qui fut en relation avec Larrey. On peut suivre Chastel Pierre faisant ses études de médecine, jusqu'en 1792 aux 'Écoles Royales de chirurgie de Toulouse", puis médecin à l'armée des Pyrénées-Orientales, enfin à l'Hôpital Militaire de Castelnaudary. En l'an IV, il demande un passeport pour passer à La Guadeloupe, afin d'y continuer ses services comme médecin militaire. Détail curieux, parmi les signatiaires comme témoin de ce passeport, on peut lire le nom de Plohais, un des premiers industriels du coton a Toulouse. Au moment de son retour à La Guadeloupe, Pierre Chastel a 22 ans. Les études de médecine étaient courtes en ce temps-la!

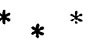

Ces papiers constituent surtout des archives familiales nous. permettant de suivre la lignée des Larchevesque-Thibaud depuis le milieu du 18e siècle jusqu'au milieu du siècle suivant. Famille bourgeoise, type même de la famille de colons à Saint-Domingue, 
vivant comme bien d'autres dans l'île, au $18 \mathrm{e}$ siècle, des revenus de leurs biens coloniaux et aussi de la judicature. Larchevesque-Thibaud père fut avocat, procureur à Saint-Domingue. Il est encore avocat à La Guadeloupe, au début du 19e siècle. Son fils aîné sera avoué à Pointe-à-Pitre. Seul, le second fils poursuivra une brillante carrière de fonctionnaire en qualité d'ingénieur de la Marine. Et bien que le rôle de constituant à Saint-Domingue et en France soit assez bien connu, ces quelques documents permettent de voir sous un jour un peu nouveau quelques aspects de la vie des LarchevesqueThibaud. On suit cette famille attachée aux Antilles pendant tout le début du $19 \mathrm{e}$ siècle et quand on sait la rareté des documents sur la Guadeloupe au début de ce siècle, on ne peut que se féliciter de l'aubaine de posséder des documents nouveaux.

Cet essai n'est qu'un premier sondage, une première ébauche après inventaire. La méditation sur les textes est nécessaire. La question de savoir si tous ces textes sont inconnus et inédits, étant résolue, il sera peut-être possible de tenter un nouveau portrait de Larchevesque-Thibaud, le Constituant. La rectification de certaines assertions malveillantes de Garran-Coulon s'imposera alors... Parler de réhabilitation serait peut-être un peu osé.

On se réjouira enfin de voir une famille de la bourgeoisie coloniale continuer une tradition...

\section{Les papiers de La Lanne Mirless}

Ces papiers constituent également des archives de famille reliées en trois forts volumes et appartenant à $M$. Robin de La Lanne Mirless (12, Basil Mansions - Basil Street, London, S.W.3. Angleterre).

M. de La Lanne Mirless, de nationalité anglaise, a recueilli les différents documents provenant d'un de ses ancêtres, PeyriguéLalanne, natif de Labatut en Bigorre. Peyrigué Lalanne avait émigré à Saint-Domingue, au 18e siècle, il avait été gérant d'une caféière à La Marmelade dans le nord de l'île et il avait réussi à acquérir une petite plantation. Il avait été un exemple pour de nombreux compatriotes qui, également originaires de Labatut, 
étaient venus à Saint-Domingue le rejoindre et s'installer dans le même quartier.

Ces papiers qui comprennent des lettres, quelques états de plantations pour deux ou trois années seulement, des états d'esclaves avant 1789 , des factures de marchandises, des papiers divers dont deux lettres de généraux haut-pyrénéens, le Général Noguès et le generral Rey qui furent aussi aux Antilles. Cette documentation reste assez disparate et fragmentaire; les papiers sont reliés chronologiquement et sans méthode.

L'intérêt de ces archives est de nous donner les noms de nombreux bigourdans ou gens de Rivière-Basse, des Quatre Vallées, d'Armagnac, passés à Saint-Domingue, au 18e siècle. Une trentaine de noms peuvent ainsi être relevés. Ces bigourdans appartenaient soit à la noblesse rurale, soit à la bourgeoisie; il y avait aussi des artisans spécialisés. La plupart sont originaires de Labatut, de Castelnau-Rivière-Basse, de Marciac, de Plaisance. Presque tous sont fixés et groupés dans le nord de l'île, dans la région du Hautdu-Trou-du-Dondon. Presque tous aussi établiront des caféières. Certains états permettent d'avoir au moins en partie un aspect de ces plantations, petites plantations puisque possédant de 12 à 37 nègres et types même de la plantation créé à force d'économies et de travail. Parfois les limites, les abornements sont indiqués. Quelques lettres relatives à la période 1791-1793 permettent de voir la situation dans le nord de l'île. Après l'incendie du Cap, la plupart des bigourdans sont retournés en France. Ils reçoivent quelques lettres de leurs compatriotes demeurés dans l'île. Peyrigué-Lalanne, après un séjour en France, retourne en 1798 avec l'escorte du général Hédouville, à Saint-Domingue. Enfin, quand tout est perdu, il passe à Philadelphie où il retrouve d'autres colons émigrés. D'Amérique, il s'occupera pour lui et ses compatriotes de l'indemnité à toucher et plus heureux que beaucoup d'entre eux, il réalisera une certaine fortune.

Ainsi ces archives familiales qui durent être plus importantes et que le dernier descendant des Peyrigué-Lalanne a précieusement conservées permettent de donner une assez vaste fresque du mouvement bigourdan à Saint-Domingue au $18 \mathrm{e}$ siècle et permettent aussi de reconstituer un moment de l'histoire antillaise. 


\section{LeS PAPIERS Th̀̀Ze}

Ces papiers appartiennent à la famille Bouvet, de Camalèsen-Bigorre. Ils forment un petit dossier qui est un inventaire des biens de Jean de Thèze, à Saint-Domingue. Cet inventaire a été dressé le 8 décembre 1792, en présence de deux experts, d'un surexpert, d'un notaire. Jean de Thèze est mort, en 1791, ou au début de 1792, après son retour de France à Saint-Domingue et après l'insurrection des noirs et la dévastation de cette partie de l'île. Il était né à Camalès-en-Bigorre, et il était propriétaire d'une caféière Saut-des-Pins, au Petit-Trou. Il fut député du Petit-Trou, à la première assemblée coloniale et un des 85 députés à venir plaider leur cause en France.

L'inventaire ne donne pas la date de son arrivée à Saint-Domingue. Nous savons seulement qu'il fut arpenteur avant d'être colon.

Cette famille bigourdane des Thèze était alliée avec les Bouvet, bigourdans aussi et qui allèrent également à Saint-Domingue. Une famille de colons bigourdans, sans aucun doute, puisque appartenant à la ligne maternelle des Bouvet, on peut ajouter les Salaignac et les Lataste propriétaires dans l'île. Madame de Thèze était une Lataste.

L'inventaire est assez précis. Il y a une description des bâtiments qui donne l'impression d'une très moyenne plantation; l'indication des cultures, les pieds de café sont estimés sur pied, chose assez rare. Les limites de la plantation, les abornements sont donnés. Au total, il y a 132 carreaux de terre. Le chiffre de la main-d'œuvre est de 39 nègres; le nombre des animaux ne figure pas dans l'inventaire. La plantation atteint une valeur de 205.790 livres, 10 sols. L'inventaire indique également qu'aucun papier, hardes, linges ou meubles ne restent, le tout ayant été pillé ou incendié.

Cet inventaire isolé peut être utilisé comme élément de comparaison avec d'autres inventaires. Il donne une image assez nette d'une plantation à Saint-Domingue et c'est en multipliant l'analyse de ces documents qu'il sera possible d'avoir une vue plus précise de certains quartiers. Inventaires rencontrés dans les archives privées, dépouillement des minutes des notaires contribueront à restituer l'aspect du Saint-Domingue du XVIIIe siècle. 
Tout en indiquant sommairement la provenance de ces papiers et en donnant un aperçu de leur nature, (lettres, inventaires, mémoires, comptes, actes not́́riés, etc...) nous avons surtout cherché à mettre en lumière les problèmes les plus saillants de chaque dossier; plus qu'archivistes, nous avons voulu être historiens. Tantôt, il s'agit des changements de générations à Saint-Domingue, tantôt de l'absentéisme, tantôt de l'esclavage, tantôt du rapport de telle ou telle culture (sucre, café, etc...) tantôt d'une classe sociale, de l'émigration dans l'île au XVIIIe siècle, tantôt de faits politiques, souvent de questions économiques et sociales.

Dans chaque dossier, nous avons cherché à mettre l'accent sur le problème qui paraissait central, estompant volontairement les autres et réservant les développements à une étude approfondie.

\section{Roger Massio,}

Professeur au Collège Vic-Bigorre (H.-P.), France. 